\title{
Electrokinetic Power Generation from Liquid Water Microjets
}

\author{
Andrew M. Duffin and Richard J. Saykally* \\ Department of Chemistry, University of California, Berkeley, CA 94720 \\ And
}

Chemical Sciences Division, Lawrence Berkeley National Laboratory, Berkeley, CA 94618

* Author to whom correspondence should be addressed.

Email: $\quad$ saykally@berkeley.edu

Address: Department of Chemistry

University of California at Berkeley

Berkeley, CA 94720

Phone: $\quad$ (510) $642-8269$ 


\begin{abstract}
Although electrokinetic effects are not new, only recently have they been investigated for possible use in energy conversion devices. We have recently reported the electrokinetic generation of molecular hydrogen from rapidly flowing liquid water microjets [Duffin et al. $J P C$ $C$ 2007, 111, 12031]. Here, we describe the use of liquid water microjets for direct conversion of electrokinetic energy to electrical power. Previous studies of electrokinetic power production have reported low efficiencies $(\sim 3 \%)$, limited by back conduction of ions at the surface and in the bulk liquid. Liquid microjets eliminate energy dissipation due to back conduction and, measuring only at the jet target, yield conversion efficiencies exceeding $10 \%$.
\end{abstract}

\title{
Keywords
}

streaming current, streaming potential, microchannel, electrical double layer 


\section{Introduction}

A central goal of current energy research is to efficiently produce electricity from renewable sources. Recently, the energy conversion properties of micro- and nanofluidic devices have received increased attention. ${ }^{1}$ A number of studies have focused on producing electrokinetic currents by forcing water through porous materials. ${ }^{2-4}$ Others have concentrated on the electrical properties of single well-defined channels. ${ }^{5-10}$ Similarly, in a recent paper, we demonstrated intrinsic electrokinetic molecular hydrogen production by flowing pure water through metal microchannels. ${ }^{11}$

Whether via multiple pores or a single channel, electrokinetic current generation depends on the overlap of a fluid velocity profile and the anisotropic charge distribution existing near a solid-liquid interface. The charge distribution at the surface, or electrical double layer, forms as certain ions in solution preferentially adsorb to a neutral or charged solid surface. This preferential interaction of ions with the surface induces counter ions of the opposite charge to redistribute near the interface. When tangential fluid flow overlaps significantly with the electrical double layer, unbalanced charges are carried downstream. More precisely, the electrokinetic streaming current can be calculated by equating it with the product of the charge density distribution, $\rho(r)$, and the fluid velocity profile, $v(r)$, integrated over the interface..$^{7-9,11-14}$ For example, the streaming current from a circular channel is given by

$$
I_{s}=2 \pi R \int_{0}^{R} v(r) \rho(r) d r,
$$

where $\mathrm{R}$ is the channel radius and $\mathrm{r}$ is the distance from the channel wall.

For non-overlapping double layers, the charge density distribution is typically represented using a Poisson-Boltzmann description. ${ }^{6}$ For micro and nanofluidic devices, the velocity profile is usually modeled by Poiseuille flow ${ }^{6}$; however, for short channels dominated 
by entrance effects, an undeveloped "top hat" model is more appropriate. ${ }^{11}$ Poiseuille flow leads to streaming currents that vary linearly with the fluid velocity, while undeveloped flow leads to streaming currents that increase nearly quadratically with flow rate. For the latter case, the streaming current is effectively fit with the equation ${ }^{11}$

$$
I_{s}=\frac{-2 \pi R \varepsilon \bar{v} \zeta}{\delta x},
$$

where $\bar{v}$ is the average fluid velocity, $\delta \mathrm{x}$ is a measure of the laminar sublayer thickness $\left(\delta \mathrm{x}=116 \cdot \mathrm{R} \cdot \mathrm{R}_{\mathrm{e}}^{(-7 / 8)}\right), \varepsilon$ is the permittivity of the medium times the permittivity of free space, and $\zeta$ is the potential at the shear plane. $R_{\mathrm{e}}$ is the Reynolds number.

To date, efforts at using streaming currents to generate electrical power have employed two reservoirs connected by means of a single channel or porous material. Initially, high pressure is applied to one reservoir and charge is moved across the channel, building up in the receiving reservoir. As this polarization evolves, the unbalanced charges in the two reservoirs create a streaming potential and the streaming potential drives ions against the fluid flow. At equilibrium, the net current is zero and back conduction exactly equals the streaming current. A low resistance path between the two reservoirs, or between each reservoir and electrical ground, short circuits back conduction and can be used to measure the streaming current. Alternatively, a high resistance probe inserted between the reservoirs can be used to measure the streaming potential. The streaming potential, together with the streaming current, ultimately determine the electrical power that can be generated from the device. When a load resistor is placed between the reservoirs, it becomes straightforward to calculate the energy conversion efficiency, $\eta$, as energy output divided by energy input, or in this case, electrical power divided by volumetric flow rate and pressure differential, 


$$
\eta=\frac{I_{s}{ }^{2} R_{L}}{Q \Delta P}
$$

Here $I_{S}$ is the streaming current, $R_{L}$ is the resistance, $Q$ is the volumetric flow rate, and $\Delta P$ is the pressure drop across the channel. Notwithstanding calculations predicting efficiencies as high as $15 \%,{ }^{6,8,15}$ experiments have thus far yielded efficiencies of only $3.2 \%^{7}$ and $0.80 \%{ }^{3}$ for a single nanochannel and a porous glass plug, respectively.

Low energy conversion efficiency in previous experiments is attributed to surface back conduction of ions. ${ }^{7,16,17}$ Electrokinetic current generation, like all electrokinetic effects, relies on an anisotropic ion distribution at an interface. Ions at the solid surface engender surface conduction and provide an additional route for dissipating charge, reducing conversion efficiency. Electrokinetic power generation using liquid water microjets eliminates both surface and bulk back conduction via creation of a jet of water that breaks up into a droplet train before reaching the receiving reservoir. Under these conditions, accumulated charge can only dissipate through the load resistor and efficiency is dramatically increased. In addition, the thin metal jet orifice creates flow conditions wherein entrance effects dominate and, consequently, the streaming current increases nearly quadratically with flow rate.

\section{Experimental}

Liquid water microjets are produced by pressurizing water behind a thin metal orifice. Figure 1 presents the experimental design, as well as an enlarged view of the interface, illustrating the electrokinetic charge separation process. The jet orifice is a Pt-Ir electron microscope aperture (Ted Pella Inc.) that is pressed between two stainless steel plates. Clean water (18.2 $\mathrm{M} \Omega \mathrm{cm}$, Millipore Milli-Q filtered) is nitrogen-purged and vacuum degassed prior to being pressurized and forced through the aperture with a Jasco PU-2089 HPLC pump. Jet 
velocity is controlled by changing the volumetric flow rate at the pump (velocity $(\mathrm{m} / \mathrm{s})=$ flow

rate $\left(\mathrm{m}^{3} / \mathrm{s}\right) /$ area $\left.\left(\mathrm{m}^{2}\right)\right)$. The pump also measures the backing pressure and this pressure is used in Bernoulli's equation to calculate the jet velocity. The diameter of the jet is determined by matching the velocities calculated from these two methods.

Streaming currents are measured at both the jet nozzle and at a downstream copper plate (2-10 cm from nozzle) that serves as the jet target. At the nozzle, the current, $\mathrm{I}_{\mathrm{s}}$, is fed into a Keithley 428 current amplifier and the resulting signal is recorded by a computer. Current at the target, $\mathrm{I}_{\mathrm{L}}$, is recorded in the same manner, with the addition of a variable resistor, $\mathrm{R}_{\mathrm{L}}(0-200$ Gohms) before the amplifier. Both the nozzle and target are insulated from all other electrical contacts with protective Teflon sheets. For efficiency calculations, the backing pressure and volumetric flow rate from the pump are also recorded as the resistance is stepped from 0 to 200 Gohms in increments of 10 Gohms. This process is repeated at a variety of flow rates and for three different aperture diameters.

\section{Results and Discussion}

The apertures used in the experiments were determined to be 5.6, 10.3, and $19.1 \mu \mathrm{m}$ in diameter and were all within the $1 \mu \mathrm{m}$ diameter tolerance from the manufacturer. Hereafter, the apertures are referred to by their nominal diameters of 5,10 , and $20 \mu \mathrm{m}$, respectively. Figure 2 gives the streaming current data measured at the nozzle for each aperture. As a result of the undeveloped flow conditions in the aperture, there is a nearly quadratic increase in streaming current with increasing fluid velocity. In addition, there is a clear increase in the magnitude of the streaming current as a function of jet diameter. This increase is proportional to the increase in metal-water interfacial surface area, i.e. aperture circumference. 
The solid lines in the figure are the best fits to the data using Equation 2, with $\zeta$ as the only adjustable parameter. Equation 2 yields zeta potentials of $-0.108,-0.135$, and $-0.105 \mathrm{~V}$ for the 5,10 , and $20 \mu \mathrm{m}$ jets, respectively. The accuracy of these measured zeta potentials depends not only on the use of correct aperture diameters, but also on the proper application of doublelayer and flow-profile theory. Even with the simple models used to develop Equation 2, the excellent fit to the data offers self consistent evidence for the accuracy of both the aperture sizes as well as the zeta potentials themselves. Accordingly, the average of $-0.12 \pm 0.02 \mathrm{~V}$ is a reasonable estimate of the potential at the shear plane of the water-Pt interface.

In traditional streaming current experiments, the conductive nature of metals gives rise to Faradaic depolarization currents that can cause inconsistencies when measuring electrokinetic properties. ${ }^{18}$ Oosterman ${ }^{19}$ found the streaming currents from platinum to give particularly irreproducible results and "the tentative explanation correctly referred to the need for controlling the discharge of protons and hydroxyl ions..."18 Streaming currents from liquid microjets eliminate or minimize Faradaic depolarization currents and give spatial control over the discharge of protons and hydroxide ions. Consequently, liquid microjet techniques may prove to offer accurate measurements of electrokinetic properties for conducting materials.

It should be noted that the signs of the currents imply that negative ions discharge at the nozzle interface while positive ions travel downstream and discharge at the target. Ostensibly due to their large inductive interactions, anions are known to specifically absorb to electrode surfaces. When pure water is used as the electrolyte, auto-dissociated hydroxide ions absorb to the nozzle interface while the associated hydrated protons form the diffuse portion of the double layer. It should also be noted that although Pt-Ir apertures were used, Molybdenum apertures give similar currents and it is unlikely that catalytic effects are responsible for the current 
generation process. Previous experiments indicate that metals have similar electrokinetic charge generation rates. ${ }^{13}$ Increasing the nozzle temperature as well as adding electrolytes decreased the electrokinetic charge generation rate.

Tangential fluid flow shears the hydrated protons from the surface and leaves unbalanced hydroxide ions at the metal-water interface. Excess, unbalanced charge at the interface induces the hydroxide ions to discharge into the metal and the observed current at the nozzle may be generated according to the process

$$
2 \mathrm{OH}^{-} \rightarrow \mathrm{H}_{2} \mathrm{O}+1 / 2 \mathrm{O}_{2}+2 e^{-}
$$

Similarly, the liquid jet is enriched in unbalanced hydrated protons that extract electrons from the jet target electrode. We have recently shown that reduction of the protons at the metal target leads to production of molecular hydrogen. ${ }^{11}$

$$
2 \mathrm{H}^{+}+2 e^{-} \rightarrow \mathrm{H}_{2}
$$

When there is no added resistance in the target circuit, the current measured at the target is always equal in magnitude and opposite in sign to the current at the nozzle; that is, when $\mathrm{R}_{\mathrm{L}}=$ $0, \mathrm{I}_{\mathrm{S}}=\mathrm{I}_{\mathrm{L}}$. For a given aperture and flow rate, the nozzle current or the current at the target at zero resistance represent the maximum amount of charge available for energy conversion. Figure 3 is a bar plot and a line plot of $\mathrm{I}_{\mathrm{L}}$ for the $20 \mu \mathrm{m}$ jet as a function of flow rate and resistance. The sign of the target current has been inverted for clarity. $\mathrm{I}_{\mathrm{s}}$, data shown in Figure 2, was collected simultaneously and was invariant to changes in resistance. The constant current at the nozzle suggests that external fields from the target reservoir do not interfere with the charge generation process and are not responsible for the decrease in $\mathrm{I}_{\mathrm{L}}$ with $\mathrm{R}_{\mathrm{L}}$.

The lower panels of Figure 3 display the calculated voltage across the load resistor. A close inspection of Figure 3 reveals that, for the two lowest flow rates, the current remains 
constant while the voltage increases linearly as a function $\mathrm{R}_{\mathrm{L}}$. Physically, protons from the liquid jet discharge at the target reservoir. As the resistance to the flow of charge increases, the target reservoir collects more hydrated protons and the voltage increases. In accordance with Ohm's law, the increased voltage compensates for the increased resistance and the current remains constant. For the higher flow rates, the linearity of Ohm's law breaks down and the voltage increases nonlinearly until a maximum of $\sim 23 \mathrm{kV}$ is reached. At these higher flow rates, charge is added to the target reservoir faster than it can dissipate through the load resistor. Under these conditions, the reservoir becomes 'filled' with charge and the voltage reaches a maximum. When the maximum voltage is reached, the current decreases linearly with increasing resistance.

The decreasing current at the target in conjunction with the constant current at the nozzle necessitates the existence of an alternate charge dissipation path that becomes available at high voltages. That is, when the reservoir becomes 'filled,' charge can leak out, possibly by the ionization of ambient air or conduction along the surface of the receiving vessel. Figure 4 shows a possible circuit diagram that includes an alternate dissipation path in terms of a system resistance, $\mathrm{R}_{\mathrm{sys}}$, and current, $\mathrm{I}_{\text {sys. }}$ In other words, as the charge/voltage at the target increases, new dissipation pathways become available and/or alternate channels become more favorable. Consequently, $\mathrm{R}_{\text {sys }}$ is a complicated function of $\mathrm{R}_{\mathrm{L}}$. Moreover, the new dissipation pathways seem to limit the maximum voltage to $\sim 23 \mathrm{kV}$, although it may be possible to increase this value with better insulation techniques. To maintain charge neutrality, the system current and load current, $\mathrm{I}_{\mathrm{L}}$, must sum to the constant nozzle current, $\mathrm{I}_{\mathrm{L}}+\mathrm{I}_{\mathrm{sys}}=\mathrm{I}_{\mathrm{s}}$.

Despite the power losses in the system, knowledge of $\mathrm{I}_{\mathrm{L}}$ and $\mathrm{R}_{\mathrm{L}}$ permit straightforward calculations of power generation and conversion efficiency. Figure 5 shows the plots of both power and efficiency for the three jet diameters measured as a function of flow rate and load 
resistance. The power scales directly with resistance and with the square of the current. Consequently, for the lower flow rates, where the current remains constant, the power increases linearly with resistance. At higher flow rates, the decreasing current competes with the increasing resistance and for each flow rate the power reaches a maximum. Additionally, the power production is related to the amount of charge that can be separated in the nozzle and, as a result, the peak power should scale with the aperture diameter.

Figure 6 plots the peak power for the three apertures and confirms not only that the highest power is obtained with the largest aperture, but also that there is a linear relationship (correlation coefficient, $\mathrm{r}=0.999$ ) between peak power production and aperture diameter. Despite the fact that the peak values were found at different velocities and resistances, the linear relationship suggests that the maximum power obtainable for an aperture is directly related to the metal-water interfacial surface area.

The efficiency plots, lower panels in Figure 5, show the same functional form as the power plots, only weighted towards the lower flow rates. The efficiency is simply the power divided by the volumetric flow rate and backing pressure. At a given flow rate, the pressure remains constant and, consequently, the efficiency has the same form as the power, simply scaled according to the flow rate and pressure. As a result, the efficiency involves a competition between the increase in power with aperture size and the corresponding increase in flow rate. Increases in channel diameter necessitate increases in flow rate that scale with the open aperture area (area $\propto$ diameter $^{2}$ ). Consequently, the larger diameter apertures require inordinately larger flow rates to obtain the associated increases in power. This leads to a decrease in efficiency, as a larger fraction of the hydrodynamic driving power is "wasted". Figure 6 also plots the peak efficiency for the three jet diameters. The peak efficiency increases along with peak power when 
going from the $5 \mu \mathrm{m}$ jet to the $10 \mu \mathrm{m}$ jet, but decreases upon moving to the $20 \mu \mathrm{m}$ jet due to the associated increase in flow rate. For the limited number of jet diameters measured in this study, a maximum efficiency of $10.7 \%$ is obtained from a $10 \mu \mathrm{m}$ diameter jet.

The metal aperture yields a current that is always equal in magnitude and opposite in sign to the current at the downstream target. In addition, separate observations have shown that the current at the target is independent of whether the nozzle is electrically grounded or floating, i.e. voltage can build up on the jet nozzle without affecting the current generation process. These observations imply that resistors placed in the nozzle circuit may be used to create additional electrical energy without affecting the conversion process at the target and without requiring additional mechanical input energy. In addition, further increases in efficiency may be realized by maximizing surface area at the expense of cross sectional area, i.e. rectangular jets.

The electronic properties of liquid water microjets create what is essentially a high voltage, low current battery. ${ }^{2}$ Although the corresponding circuit diagram is useful, it should be remembered that the current generation technique is unusual. In a battery or photovoltaic cell, open circuit conditions will increase the potential and eventually halt the current generation process. In contrast, an open circuit does not affect the charge separation process in a liquid microjet and the maximum voltage should only be limited by the ability of the receiving vessel to hold unbalanced charge. In this way, the voltage that drives the energy conversion process could be used separately for the upstream and downstream circuits. In addition, it may be possible to convert the current and employ a simple step-down transformer to obtain more useable voltages and currents.

\section{Conclusions}


Liquid microjets, created by forcing water through a small metal orifice, offer marked advantages over the use of channels and porous materials for electrokinetic power generation. By creating a jet of water that breaks up (via Rayleigh instabilities) before reaching the receiving reservoir, both surface conduction and bulk conduction, which otherwise limit conversion efficiency, are eliminated. In addition, the thin metal orifice creates flow conditions wherein entrance effects dominate. Consequently, the streaming current increases nearly quadratically with flow rate, whereas the laminar flow conditions obtained in channels and porous plugs affect only a linear current increase with flow rate. Using a liquid microjet, accumulated charge can only dissipate through the load resistor, and conversion efficiency is significantly increased with respect to channels and porous plugs. However, at high load resistance, sufficient voltage is produced to allow additional dissipation pathways. Despite these high voltage leakage currents, conversion efficiencies above $10 \%$ can easily be realized with liquid microjets, and considerable higher values should be achievable.

\section{Acknowledgements}

This work was supported by the Director, Office of Science, Office of Basic Energy

Sciences, of the U.S. Department of Energy under contract no. DE-AC02-05CH11231. We thank Dorian Liepmann in the UCB Bioengineering Department and Henry Chan in the UCB Chemistry Department for helpful conversations. 


\section{References}

(1) Pennathur, S.; Eijkel, J. C. T.; van den Berg, A. Lab on a Chip 2007, 7, 1234.

(2) Yang, J.; Lu, F. Z.; Kostiuk, L. W.; Kwok, D. Y. Journal of Micromechanics and Microengineering 2003, 13, 963.

(3) Yang, J.; Lu, F. Z.; Kostiuk, L. W.; Kwok, D. Y. Journal of Nanoscience and Nanotechnology 2005, 5, 648.

(4) Olthuis, W.; Schippers, B.; Eijkel, J.; van den Berg, A. Sensors and Actuators BChemical 2005, 111, 385 .

(5) van der Heyden, F. H. J.; Stein, D.; Dekker, C. Physical Review Letters 2005, 95.

(6) van der Heyden, F. H. J.; Bonthuis, D. J.; Stein, D.; Meyer, C.; Dekker, C. Nano Letters 2006, 6, 2232.

(7) van der Heyden, F. H. J.; Bonthuis, D. J.; Stein, D.; Meyer, C.; Dekker, C. Nano Letters 2007, 7, 1022.

(8) Daiguji, H.; Yang, P. D.; Szeri, A. J.; Majumdar, A. Nano Letters 2004, 4, 2315.

(9) Werner, C.; Korber, H.; Zimmermann, R.; Dukhin, S.; Jacobasch, H. J. Journal of Colloid and Interface Science 1998, 208, 329.

(10) Morrison, F. A.; Osterle, J. F. Journal of Chemical Physics 1965, 43, 2111.

(11) Duffin, A. M.; Saykally, R. J. Journal of Physical Chemistry C 2007, 111, 12031.

(12) Rice, C. L.; Whitehead, R. Journal of Physical Chemistry 1965, 69, 4017.

(13) Gavis, J.; Koszman, I. Journal of Colloid Science 1961, 16, 375.

(14) Delgado, A. V.; Gonzalez-Caballero, F.; Hunter, R. J.; Koopal, L. K.; Lyklema, J. Journal of Colloid and Interface Science

Elkin 06, International Electrokinetics Conference, June 25-29, Nancy, France 2007, 309, 194.

(15) Min, J. Y.; Hasselbrink, E. F.; Kim, S. J. Sensors and Actuators B-Chemical 2004, $98,368$.

(16) Lyklema, J. Journal of Physics-Condensed Matter 2001, 13, 5027.

(17) Davidson, C.; Xuan, X. C. Electrophoresis 2008, 29, 1125.

(18) Duval, J. F. L.; Huijs, G. K.; Threels, W. F.; Lyklema, J.; van Leeuwen, H. P. Journal of Colloid and Interface Science 2003, 260, 95.

(19) Oosterman, J. Thesis, University of Utrecht, 1937. 


\section{Figure Captions}

Figure 1: Experimental design; the expanded view shows details of the electrokinetic charge separation process

Figure 2: Data points indicate streaming current measurements, taken at the jet nozzle, for $5 \mu \mathrm{m}$ $(\mathbf{\Lambda}), 10 \mu \mathrm{m}(\mathbf{\bullet})$, and $20 \mu \mathrm{m}(\bullet)$ diameter apertures. The solid lines are the best fits to the data using Equation 2, with zeta potentials calculated to be $-0.108 \mathrm{~V},-0.135 \mathrm{~V}$, and $-0.105 \mathrm{~V}$ for each diameter, respectively.

Figure 3: A) Inverted target currents from a $20 \mu \mathrm{m}$ diameter jet measured as a function of flow rate and resistance. B) Calculated voltage at the jet target from a $20 \mu \mathrm{m}$ diameter jet as a function of $\mathrm{I}_{\mathrm{L}}, \mathrm{R}_{\mathrm{L}}$, and flow rate. For the low flow rates, voltage changes linearly with $\mathrm{R}_{\mathrm{L}}$. For higher flow rates, a maximum voltage is reached and thereafter $I_{L}$ changes linearly with $R_{L}$.

Figure 4: Equivalent circuit for power generation from an electrokinetic microjet system. $I_{L}$ and $\mathrm{R}_{\mathrm{L}}$ are the current and resistance through the load resistor used to calculate conversion power and efficiency, while $\mathrm{I}_{\mathrm{sys}}$ and $\mathrm{R}_{\mathrm{sys}}$ are the current and resistance associated with system losses.

Figure 5: Plots of power (top panels) and conversion efficiency (bottom panels) for 5, 10, and 20 $\mu \mathrm{m}$ diameter jets (left to right). Both power and efficiency are plotted as a function of flow rate and resistance. Peak power increases with increasing aperture diameter, noting that the electrical power is only generated at the metal-water interface. Peak efficiency reaches a maximum at the 
intermediate diameter due to the interplay between increased power production and increased volumetric flow rate.

Figure 6: Peak power $(\bullet)$ and energy conversion efficiency $(\Delta)$ measurements for 5,10 , and 20 $\mu \mathrm{m}$ diameter jets (Data taken from the complete plots shown in Figure 5). Peak power increases linearly with jet diameter while peak efficiency is greatest at $10 \mu \mathrm{m}$. 
Figures

Figure 1:

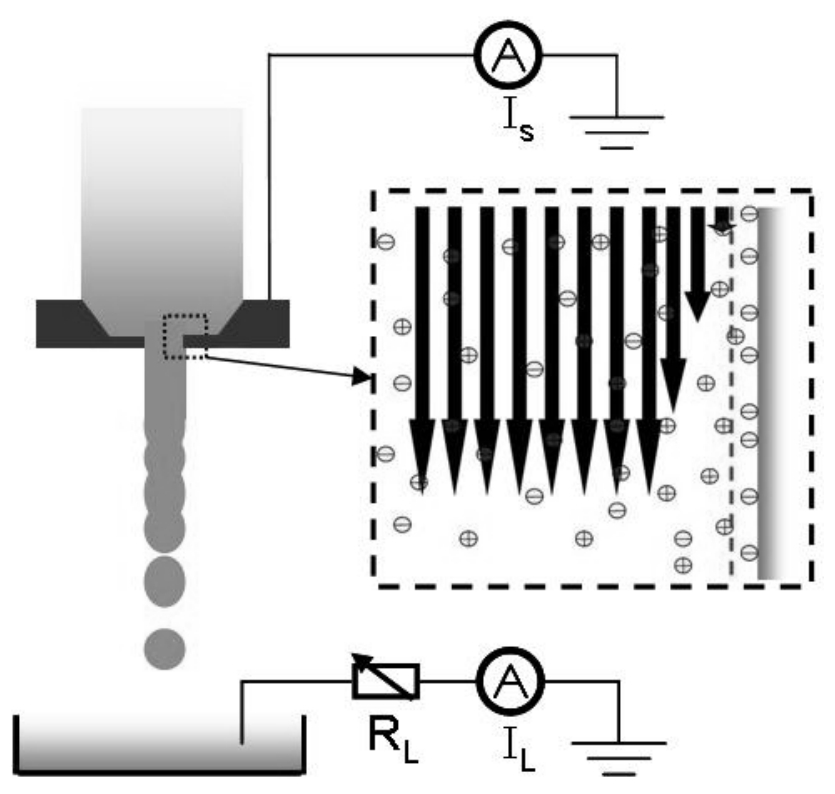

Figure 2:

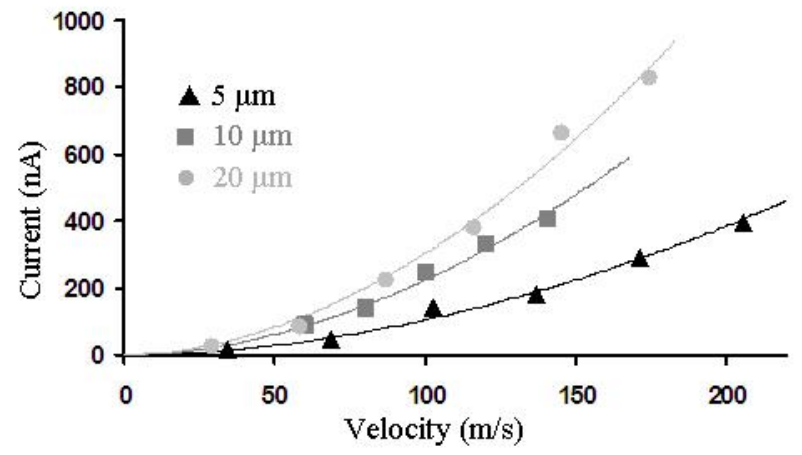


Figure 3:
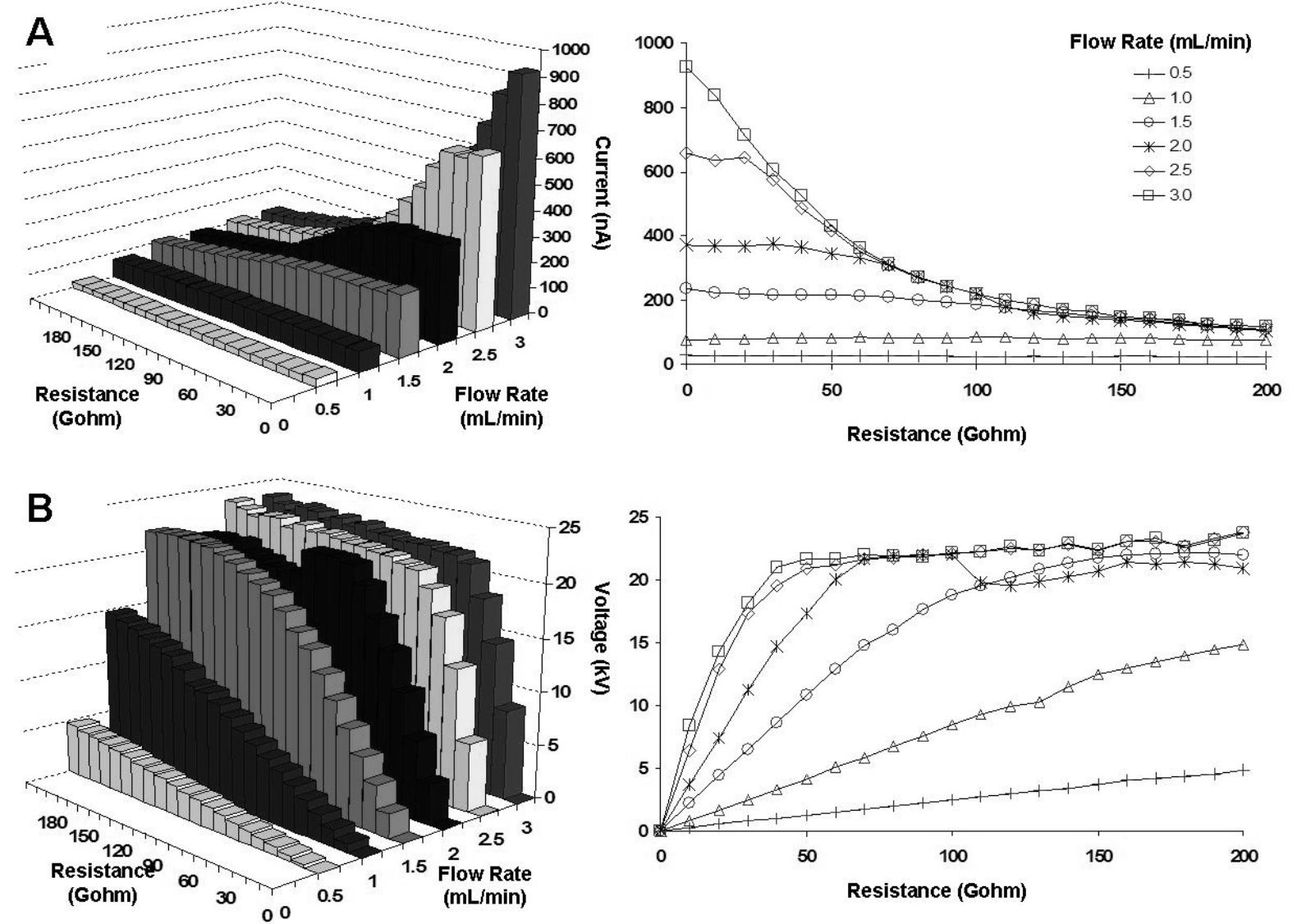

\section{Figure 4:}

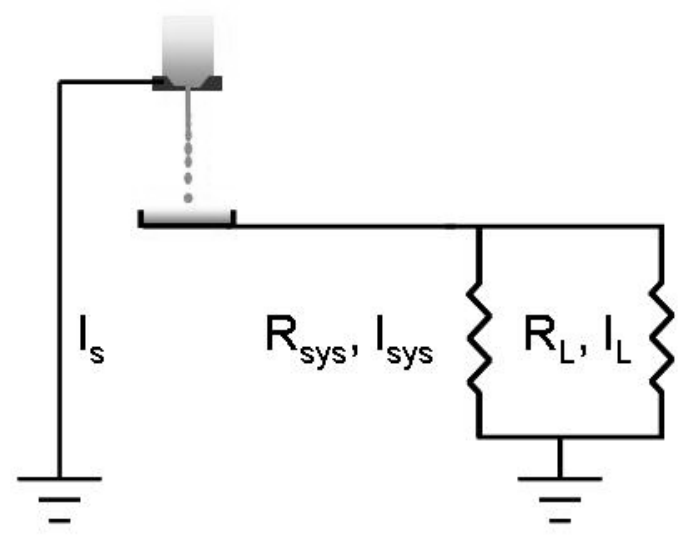


Figure 5:
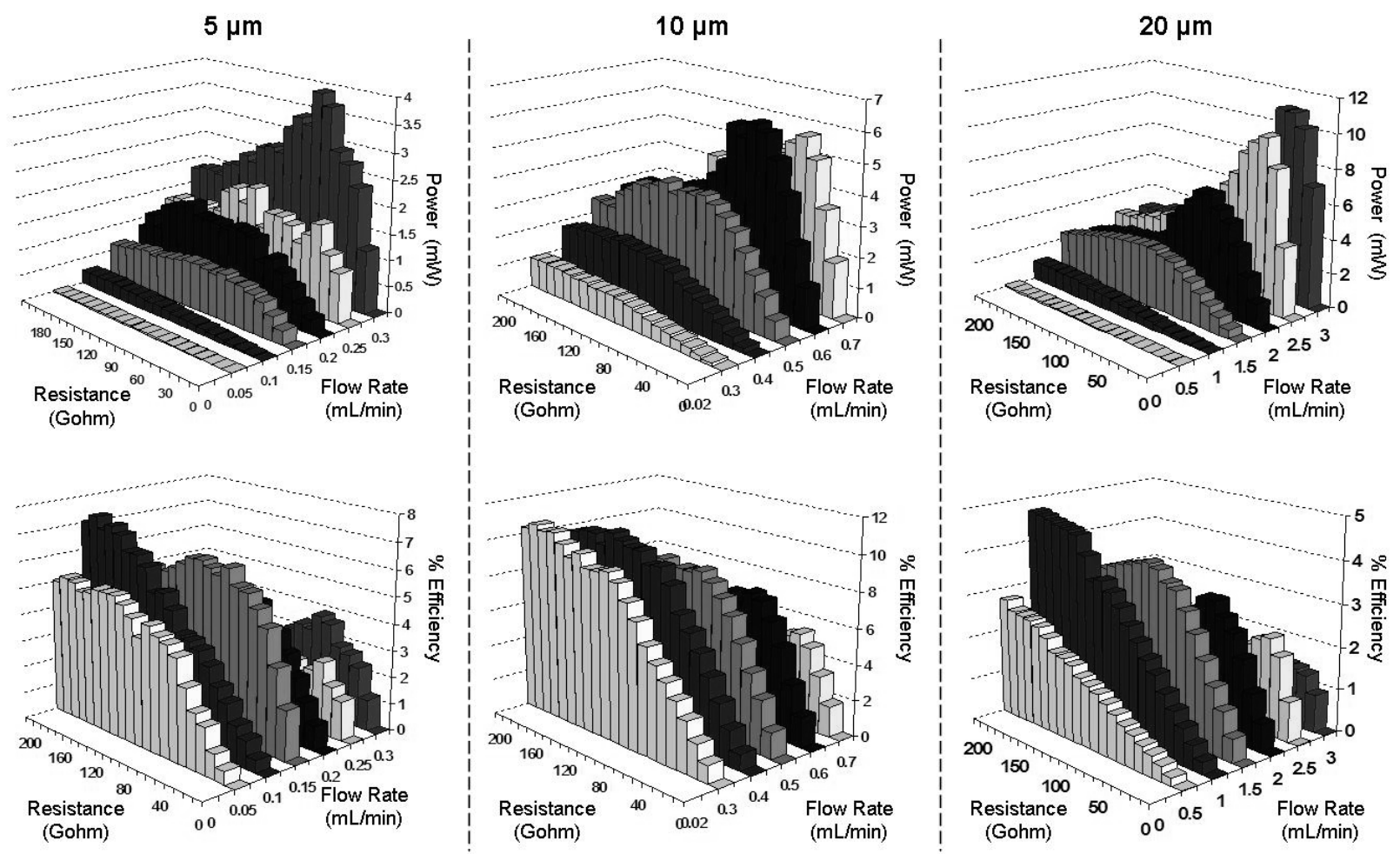

Figure 6:

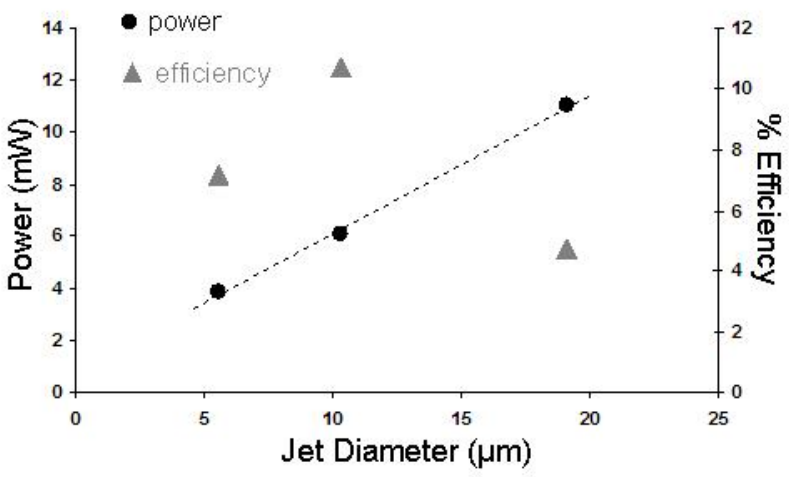

